\title{
Single Photon Emission from Site- Controlled InAs Quantum Dots Grown on GaAs(001) Patterned Substrates
}

\author{
J. Martín-Sánchez, ${ }^{+}{ }^{+*}$ G. Muñoz-Matutano, ${ }^{\ddagger}$ J. Herranz, ${ }^{\dagger}{ }^{~ J . ~ C a n e t-F e r r e r, ~}{ }^{\ddagger}$ B. Alén, ${ }^{\dagger}{ }^{\text {Y. González, }}{ }^{\dagger}$ \\ P. Alonso-González, ${ }^{\dagger}$ D. Fuster, ${ }^{\dagger}$ L. González, ${ }^{\dagger}$ J. Martínez-Pastor, ${ }^{\star}$ and F. Briones ${ }^{\dagger}$ \\ ${ }^{\dagger}$ IMM, Instituto de Microelectrónica de Madrid (CNM, CSIC), Isaac Newton 8, 28760 Tres Cantos, Madrid, Spain, and `ICMUV, Instituto de Ciencia de Materiales, \\ Universidad de Valencia, P.0. Box 2085, 46071 Valencia, Spain
}

\begin{abstract}
emiconductor quantum dots (QDs)
S are often considered ideal two-level systems with a great potential for
\end{abstract} solid-state based quantum optical information technologies. This fact has been exploited in the last years combining sophisticated fabrication and characterization techniques to demonstrate their adequacy in elementary quantum optical information devices like single and entangled photon sources, ${ }^{1,2}$ or quantum logic gates. ${ }^{3,4} \mathrm{How}$ ever, for a proper integration in real and practical devices, the future development of these technologies relies on our ability to manage accurately the individual position and size of the QDs. This achievement would open up new horizons for the integration of QDs as building blocks of scalable architectures in the quantum information field.

From the technical point of view, the InAs/GaAs(001) system is of great interest due to its compatibility with well established III-V compound semiconductor technologies. However, as it is well-known, conventional growth of self-assembled QDs leads to the formation of random spatial distributions. ${ }^{5}$ To overcome this inherent problem, the substrate surface can be patterned before growth by means of different lithographic schemes. ${ }^{6-12}$ These methods combine the natural self-assembly process (bottom-up approach) with preferential formation of the nanostructures in certain locations imposed by the pattern motifs (topdown approach).

Among the different patterning methods, local oxidation nanolithography has arisen as a straightforward approach which does not require of resists or ex-situ etching processes to produce site-controlled $\ln \mathrm{As} / \mathrm{GaAs}^{13,14}$ and $\ln \mathrm{As} / \mathrm{InP}^{15}$ QDs with

ABSTRACT We present a fabrication method to produce site-controlled and regularly spaced InAs/GaAs quantum dots for applications in quantum optical information devices. The high selectivity of our epitaxial regrowth procedure can be used to allocate the quantum dots only in positions predefined by ex-situ local oxidation atomic force nanolithography. The quantum dots obtained following this fabrication process present a high optical quality which we have evaluated by microphotoluminescence and photon correlation experiments.

KEYWORDS: quantum dots $\cdot$ patterned substrates $\cdot$ single photon emitters

nanometer resolution. Yet, its drawback, common to all lithographic techniques, is still the poor optical quality of the nanostructures grown on top of the patterned area. Because of the introduction of impurities and/or defects during the patterning process, the fabrication of site-controlled QDs with a high optical efficiency remains a challenging task where the type of initial substrate, the surface preparation, and other growth parameters must be optimized to preserve the optical properties of QDs grown in close proximity to the oxidized interface. ${ }^{16}$ In this letter, we apply such optimized procedure combining local oxidation nanolithography and molecular beam epitaxy (MBE) to fabricate sitecontrolled InAs QDs with high optical quality as demonstrated by their single QD emission and photon correlation spectra.

\section{RESULTS AND DISCUSSION}

Before the pattern was fabricated, the substrate surface roughness was improved by deposition of a $0.5 \mu \mathrm{m}$ thick GaAs layer by MBE at a growth rate $r_{\mathrm{g}}=1$ monolayer (ML) per second (substrate temperature $T_{\mathrm{s}}$ $=580^{\circ} \mathrm{C}$ and $\mathrm{V} / \mathrm{III}$ flux ratio $\sim 8$ ). This epitaxial layer (EL) was grown on commercial epiready GaAs (001) substrates after removal of the GaAs oxide by a conventional thermal process. With these growth conditions flat

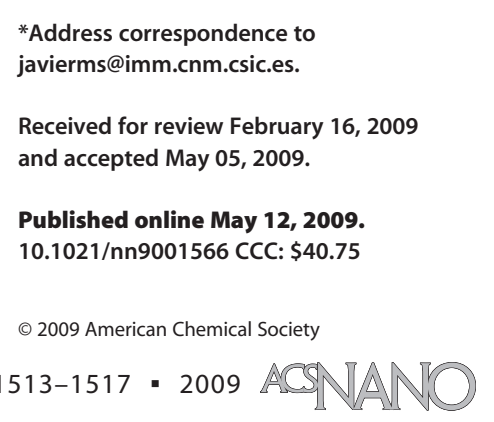




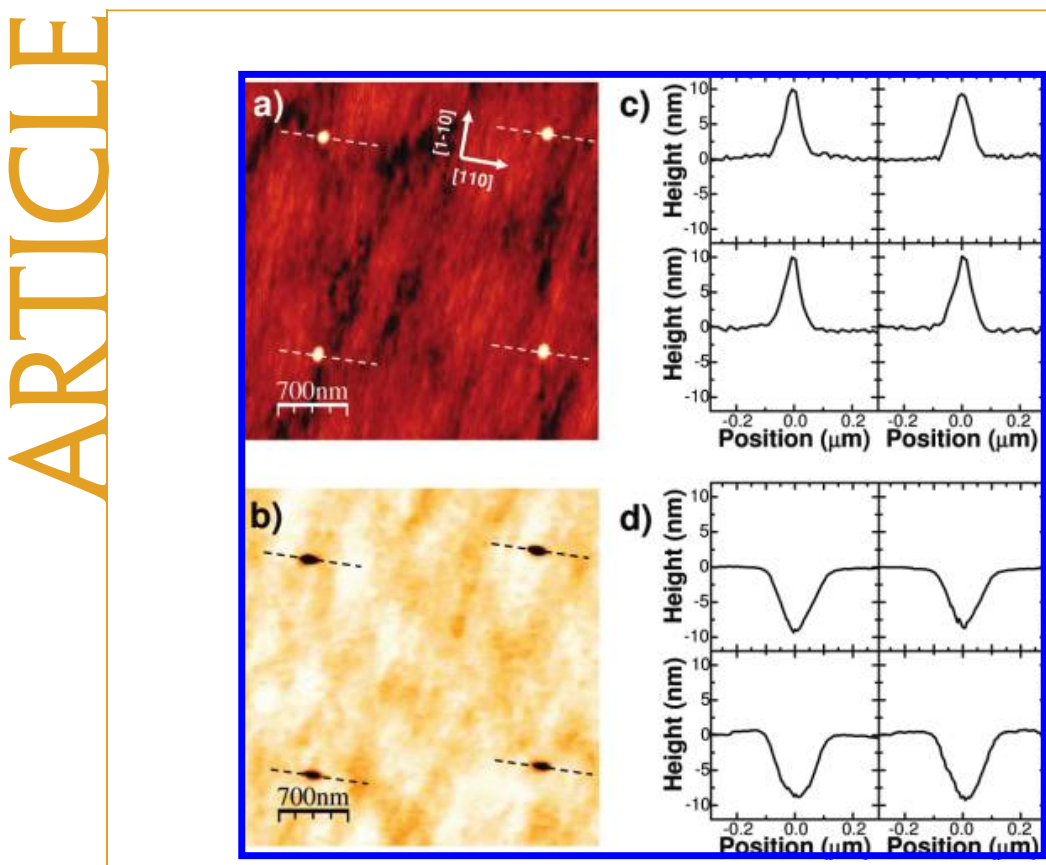

Figure 1. (a) AFM picture of the patterned area showing four GaAs oxide dots. (b) Same area after removal of the oxide dots with atomic $\mathrm{H}$ and epitaxial regrowth of a 7-nm-thick GaAs buffer layer. Corresponding profiles along the [110] crystallographic direction of the oxide dots (c) and nanoholes (d).

surfaces at the atomic level are reproducibly obtained. ${ }^{16}$ The EL substrates were then patterned by local oxidation atomic force nanolithography using a commercial Nanotec scanning probe microscopy system under controlled ambient conditions (relative humidity $\sim 30 \%$ ). ${ }^{13}$ In these experiments, the sample was set to ground and a negative bias voltage $V=-10 \mathrm{~V}$ was applied during $10 \mathrm{~s}$ to the n-doped silicon tip $(K=40 \mathrm{~N} / \mathrm{m})$ in noncontact mode. This sequence was repeated at predefined positions of the substrate to create a square matrix of GaAs oxide dots with an average width and height of $\sim 160$ and $\sim 10 \mathrm{~nm}$, respectively (Figure 1a).

The growth procedure itself started at low substrate temperature, $T_{\mathrm{s}}=490^{\circ} \mathrm{C}$, by exposing the surface to an atomic hydrogen $(\mathrm{H})$ flux during $30 \mathrm{~min}$ using a $\mathrm{Ta} \mathrm{H}_{2}$ thermal cracker with a $\mathrm{H}_{2}$ base pressure of $10^{-5}$ Torr. In addition, $\mathrm{As}_{4}$ was simultaneously supplied at a beam equivalent pressure (BEP) of $2 \times 10^{-6}$ Torr to compensate arsenic losses from the surface. A clear c(4 $\times 4)$ As rich surface reconstruction was observed on the reflection high energy electron diffraction (RHEED) pattern after 5 min exposure to atomic $\mathrm{H}$ and $\mathrm{As}_{4}$ beams, indicating that the surface oxide had been removed. At this stage, atomic force microscopy (AFM) images (not shown) reveal that the fabricated GaAs oxide dots have been also removed resulting in round nanoholes with an average width and depth of $\sim 160$ and $\sim 13 \mathrm{~nm}$, respectively. ${ }^{17}$ The aim of this work is to use these nanoholes, patterned on the GaAs surface, as preferential nucleation sites for InAs. To this respect, the large exposure time ( $30 \mathrm{~min}$ ) of the surface to $\mathrm{H}$ is a crucial step to enhance the emission efficiency of InAs QDs grown near the GaAs substrate interface. ${ }^{16}$ Previous to $\ln A s$ deposition for QDs growth, a 7-nm-thick GaAs buffer layer was grown in order to flatten the surface between patterned nanoholes. To do so, atomic layer molecular beam epitaxy (ALMBE) growth mode $\left(r_{\mathrm{g}}=0.5 \mathrm{ML} / \mathrm{s}, T_{\mathrm{s}}\right.$ $=490^{\circ} \mathrm{C}$ and BEP As $s_{4}=2 \times 10^{-6}$ Torr) was used allowing the low temperature growth of GaAs layers with flat morphologies without thickness limitation. ${ }^{18}$

The InAs deposited thickness has to be controlled to achieve a high nucleation selectivity. We have found that by using an InAs coverage below the critical value, the nucleation of self-assembled QDs in regions outside the nanoholes can be largely suppressed. For low density patterned arrays equivalent to $2.5 \times 10^{7} \mathrm{QDs} /$ $\mathrm{cm}^{2}$, we have obtained optimum results depositing 1.5 $\mathrm{ML}$ of InAs at $r_{\mathrm{g}}=0.01 \mathrm{ML} / \mathrm{s}$ and $T_{\mathrm{s}}=490^{\circ} \mathrm{C}$ with BEP $\mathrm{As}_{4}=2 \times 10^{-6}$ Torr. Under these growth conditions the GaAs surface shows a $c(4 \times 4)$ reconstruction on the RHEED diagram and the critical thickness for selfassembled InAs QDs formation on nonpatterned surfaces is $1.7 \mathrm{ML}$ of InAs. Finally, a 20-nm-thick GaAs capping layer was grown for optical investigation of the buried nanostructures. This capping layer was grown at $r_{\mathrm{g}}=0.5 \mathrm{ML} / \mathrm{s}$ in a two-step process: the first $11 \mathrm{~nm}$ at $490^{\circ} \mathrm{C}$ under BEP As $s_{4}=1 \times 10^{-6}$ Torr and the remaining $9 \mathrm{~nm}$ at $T_{\mathrm{s}}=580^{\circ} \mathrm{C}$ under BEP As $s_{4}=2 \times 10^{-6}$ Torr.

Figure 1a shows an AFM image containing several oxide dots fabricated in the lithographic step. A corresponding profile along the [110] crystallographic direction can be seen in Figure 1c. On its right, the same area has been scanned after removal of the GaAs oxide and growth of a 7-nm-thick GaAs buffer layer as explained above (Figure 1b). Although a slight elongation of the nanoholes occurs during the growth of the buffer layer, as it can be observed in (Figure 1d), the pattern motifs are preserved until the deposition of InAs. ${ }^{17}$

The AFM characterization of the patterned area after InAs deposition shows the high selectivity of our fabrication method demonstrating that the nucleation of self-assembled QDs in the nonpatterned region has been completely suppressed, obtaining only QDs formation inside the pattern motifs, as can be seen in Figure 2a. Notice that at the motifs near the edge of the patterned region, two or three QDs may be formed. This is due to the larger dimensions of the oxides fabricated at these positions on which the piezoelectric scanner drift effects are noticeable in our AFM system for the oxidation time used in this work $(10 \mathrm{~s}) .^{17}$

Figure $2 \mathrm{~b}$ shows an AFM image of a single sitecontrolled InAs/GaAs QD. The resulting nanostructures are shallow, with an average height $h=7 \pm 2 \mathrm{~nm}$ and height to diameter ratio $h / D \approx 0.08$ as shown in Figure $2 c$. The aim of our present study is to the evaluate the emission properties of the QDs formed inside the nanoholes obtained by the fabrication process explained above. To study their optical properties, a $20 \times 20 \mu \mathrm{m}^{2}$ area enclosing the patterned matrix was delimited by 


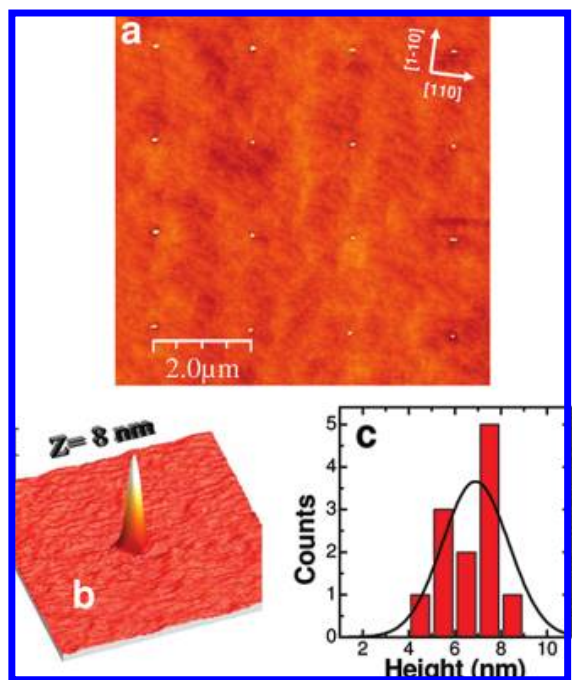

Figure 2. (a) AFM picture of a matrix containing sitecontrolled QDs with $2 \mu \mathrm{m}$ pitch period; (b) $500 \times 500 \mathrm{~nm}$ AFM picture of a single QD; (c) quantum dot height distribution before capping.

metallic markers fabricated after growth by electron beam lithography. Then, the same confocal microscope was used to find the markers and investigate the emission spectrum of the site-controlled QDs at low temperatures. In Figure 3, we show a microphotoluminescence ( $\mu-P L)$ image of the patterned area as obtained by integration of the photoluminescence in a 40-nmwide spectral window around $990 \mathrm{~nm}$. The window is broader than the average excited state splitting $\Delta E \approx$ $26 \pm 3 \mathrm{meV}$. As it can be observed, the distribution observed by AFM is reproduced with most of the sitecontrolled QDs emitting light at similar wavelengths.

The inset of Figure 4a shows a typical $\mu$-PL spectrum recorded in a region far away from the matrix. We do not find spectral features which could be associated to self-assembled quantum dots, as expected. The broad peaks around $1.360 \mathrm{eV}$ correspond to emission from the substrate associated to $\mathrm{Cu}_{\mathrm{Ga}}$ and its two longitudinal optical phonon replicas at 1.324 and 1.288 $\mathrm{eV}$, respectively..$^{19}$ Meanwhile, the peaks at 1.42 and $1.46 \mathrm{eV}$ must be associated to the emission from a wetting layer (WL) of varying thickness resulting from the deposition of InAs below the critical thickness.

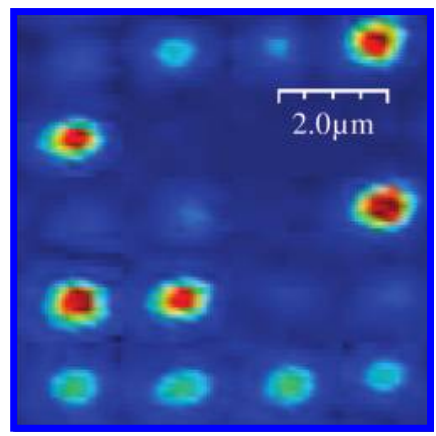

Figure 3. Spatially resolved $\mu \mathrm{PL}$ image of the site-controlled QD matrix obtained at $77 \mathrm{~K}$.

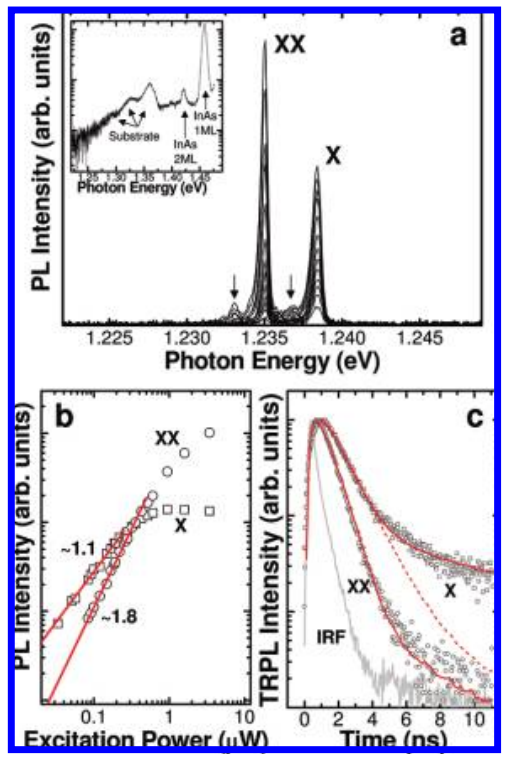

Figure 4. (a) Evolution of the $\mu \mathrm{PL}$ spectrum varying the laser excitation power from 50 to $500 \mathrm{nW}$. Inset: Emission spectrum in a region far away from the patterned area (excitation power $\sim 20 \mu \mathrm{W}$ ). (b) $\mathrm{L}-\mathrm{L}$ plot of the $\mu \mathrm{PL}$ integrated intensities for the exciton (X) and biexciton (XX) lines. The slopes of the linear fits are indicated. (c) Experimental decay curves (open symbols) for X and XX (average excitation power: $40 \mathrm{nW}$ ). Solid and dashed lines are fits to different exponential laws convoluted with the system impulse response function (IRF).

The rest of Figure 4 focuses on the $\mu \mathrm{PL}$ study of a representative site-controlled QD. Exciton (X) and biexciton $(X X)$ recombination lines can be recognized in the excitation power dependent spectra shown in Figure 4a. Their relative splitting is typical of self-assembled QDs emitting at this energy, ${ }^{20}$ as it is also the linear and superlinear dependence of their integrated intensities (Figure 4b). ${ }^{21}$ Weaker resonances, marked with down arrows in Figure $4 a$, should be related, in view of their small binding energy, to positively charged complexes associated to the impurity background and/or the presence of defects in the interface. ${ }^{21}$ Indeed, in our method, the preservation of the nanoholes during growth imposes the vicinity of a regrown interface which is usually the origin of deleterious effects in QDs. The first effect is over the emission line width which becomes inhomogeneously broadened due to spectral diffusion caused by the electrical carriers trapped at the interface. ${ }^{22}$ In our case, a Gaussian fit reproduces quite well the observed line shape with a full width at halfmaximum of $0.6 \mathrm{meV}$. The second effect is over the exciton lifetime and can compromise the emission efficiency if many nonradiative recombination channels are created at the interface. To evaluate this point, we have analyzed the time-resolved $\mu-P L$ of the exciton and biexciton resonances. The experimental decay curves are shown in Figure $4 c$ together with their best fits to exponential laws. The biexciton decay curve is well described by a single decay constant $\tau_{x x} \approx 665 \pm 20$ ps (solid line). This is approximately half of the value found 


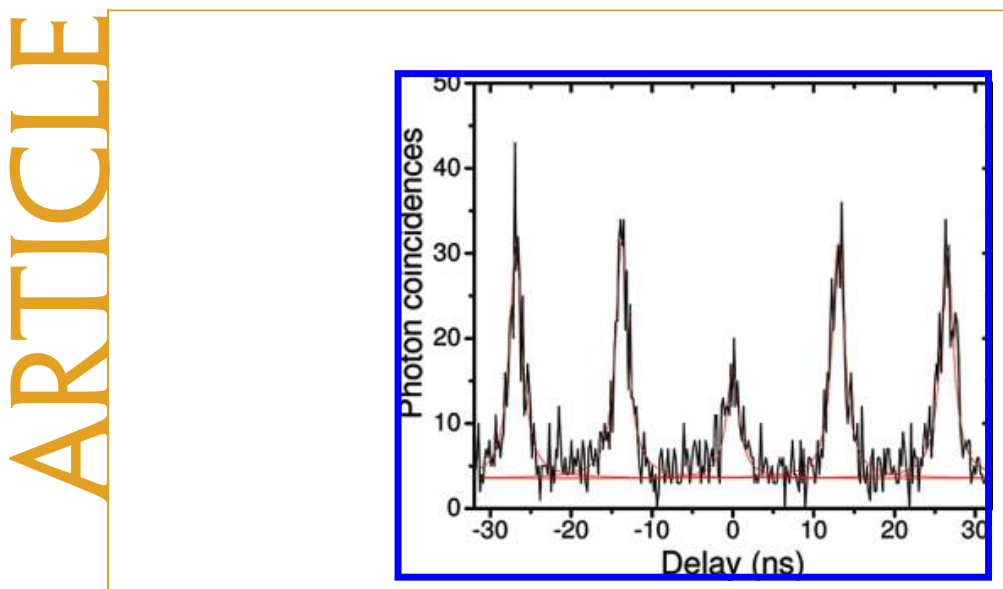

Figure 5. Photon autocorrelation measurement for a sitecontrolled QD performed at $5 \mathrm{~K}$ under average pulsed excitation power of $40 \mathrm{nW}$ at $790 \mathrm{~nm}$. The thin solid lines are Lorentzian fits used to extract the raw value of $g^{2}(0)$.

for the exciton just considering its initial decay $\left(\tau_{\mathrm{X}} \approx\right.$ $1180 \pm 40 \mathrm{ps}$, dashed line). As reported for selfassembled QDs, to fully describe the exciton decay, an additional slow component with $\tau_{\mathrm{s}} \approx 8 \pm 2$ ns must be considered (solid line). ${ }^{23}$ Nevertheless, the most important feature is the large value found for the initial decay constants. Wang et al. reported a strong decrease of the PL intensity and lifetime of QDs close to the sample surface. ${ }^{22}$ Decreasing the distance from 15 to $9 \mathrm{~nm}$, they found a QD lifetime reduction from 550 to 65 ps. In our case, the QDs and the patterned surface are separated only by $\sim 7 \mathrm{~nm}$, and still the exciton and biexciton lifetimes are those expected for self-assembled quantum dots. ${ }^{23}$ This is a satisfactory result which must be attributed to the almost complete elimination of nonradiative centers achieved using the regrowth conditions explained above.

The combination of local oxidation nanolithography and epitaxial regrowth is a powerful method to develop novel devices for quantum optical information technologies. The development of single photon sources is one of the potential applications of this process. ${ }^{1}$ Therefore, we have also measured the photon correlation statistics of our site-controlled QDs using Hanbury-Brown and Twiss interferometry. ${ }^{24}$ Figure 5 contains the autocorrelation histogram recorded for

\section{EXPERIMENTAL SECTION}

To carry out the optical characterization of the individual sitecontrolled QDs we used a diffraction limited (objective NA = 0.55 ) confocal setup working at $5 \mathrm{~K}$. As excitation source we employed a Ti-Sapphire mode-locked laser tuned to $790 \mathrm{~nm}$ and operated in continuous wave mode or subpicosecond pulsed mode at $76 \mathrm{MHz}$. A silicon charge coupled device (CCD) and a Si avalanche photodiode (APD) were attached to the same $0.3 \mathrm{~m}$ focal length spectrometer to conduct the continuous wave or time-resolved characterization of the QDs, respectively. For the photon correlation setup, the light path was divided after the spectrometer and redirected to similar APDs operating in geiger mode. Time resolved traces and photon correlation histograms were recorded with time correlated single photon counting electronics. the exciton resonance studied above and reveals the desired photon antibunching behavior at zero delay. This result shows the potential of our fabrication process in the development of quantum information devices based in site-controlled QDs. We extract a second order correlation function at zero delay $g^{2}(0)=0.46$ from integration of the data as shown in the figure. The fact that $g^{2}(0)$ is below 0.5 before correcting for our signal-to-noise ratio (SNR) indicates that multiphoton events are largely suppressed. To avoid single pulse reexcitations, the histogram was recorded reducing the excitation power as much as possible. This fact limited severely our $\mathrm{SNR} \approx 4.6$ meaning that in the best case of perfect single photon emission would lead to $g^{2}(0) \approx$ $0.32 .{ }^{25}$ The difference between the expected value and the measured value can be attributed to a long-time scale component of the carrier capture process which allows re-excitation of the single QD. ${ }^{26}$ This is supported by the presence of the slow component in the exciton decay curve and also by the observation of a vertical offset in the coincidence histogram which cannot be explained from our detector dark counts rate (signal blocked). Such behavior is typical under the above gap excitation conditions used here. ${ }^{26} \mathrm{~A}$ resonant excitation scheme could be used to investigate the role of the delayed carrier capture process which deserves further study out of the scope of this work.

\section{CONCLUSIONS}

We have presented a method to fabricate site controlled InAs/GaAs quantum dots with high optical quality using local oxidation nanolithography and molecular beam epitaxial regrowth. The nucleation selectivity of the method has been demonstrated fabricating a QD square matrix with $2 \mu \mathrm{m}$ pitch period. We have conducted an analysis of the optical properties of the individual QDs and concluded that the exciton lifetimes and overall emission properties are not compromised by the nearby patterned interface. Finally, single photon emission from site-controlled QDs has been demonstrated, validating our method for the future development of laterally arranged single photon sources.
Acknowledgment. The authors gratefully acknowledge financial support by Spanish MEC and CAM through grants NANOSELF2 (TEC-2005-05781-C03-01/03), NANINPHO-QD (TEC-200806756-C03-01/03), NANOCOMIC (S-505/ESP/000200), and Consolider-Ingenio 2010 QOIT (CSD2006-0019).

\section{REFERENCES AND NOTES}

1. Santori, C.; Pelton, M.; Solomon, G.; Dale, Y.; Yamamoto, Y. Triggered Single Photons from a Quantum Dot. Phys. Rev. Lett. 2001, 86, 1502-1505.

2. Stevenson, R. M.; Young, R. J.; Atkinson, P.; Cooper, K.; Ritchie, D. A.; Shields, A. J. A Semiconductor Source of Triggered Entangled Photon Pairs. Nature 2006, 439, 179182. 
3. Li, X.; Wu, Y.; Steel, D.; Gammon, D.; Stievater, T. H.; Katzer, D. S.; Park, D.; Piermarocchi, C.; Sham, L. J. An All-Optical Quantum Gate in a Semiconductor Quantum Dot. Science 2003, 301, 809-811.

4. Robledo, L.; Elzerman, J.; Jundt, G.; Atatüre, M.; Högele, A.; Fält, S.; Imamoglu, A. Conditional Dynamics of Interacting Quantum Dots. Science 2008, 320, 772-775.

5. Seifert, W.; Carlsson, N.; Miller, M.; Pistol, M.; Samuelson, L.; Wallenberg, L. In-Situ Growth of Quantum Dot Structures by the Stranski-Krastanow Growth Mode. Prog. Cryst. Growth Charact. 1996, 33, 423-471.

6. Heidemeyer, H.; Müller, C.; Schmidt, O. G. Highly Ordered Arrays of In (Ga)As Quantum Dots on Patterned GaAs (001) Substrates. J. Cryst. Growth 2004, 261, 444-449.

7. Alonso-González, P.; González, L.; González, Y.; Fuster, D.; Fernández-Martínez, I.; Martín-Sánchez, J.; Abelmann, L. New Process for High Optical Quality InAs Quantum Dots Grown on Patterned GaAs(001) Substrates.

Nanotechnology 2007, 18, 355-302.

8. Pelucchi, E.; Watanabe, S.; Leifer, K.; Zhu, Q.; Dwir, B.; Rios, P. D. L.; Kapon, E. Mechanism of Quantum Dot Energy Engineering by Metalorganic Vapor Phase Epitaxy on Patterned Nonplanar Substrates. Nano Lett. 2007, 7, 1282 1285.

9. Tran, T.; Muller, A.; Shih, C. K.; Wong, P. S.; Balakrishnan, G.; Nuntawong, N.; Tatebayashi, J.; Huffaker, D. L. Single Dot Spectroscopy of Site-Controlled InAs Quantum Dots Nucleated on GaAs Nanopyramids. Appl. Phys. Lett. 2007, 91, 133104-1-3.

10. Wang, L.; Rastelli, A.; Kiravittaya, S.; Atkinson, P.; Ding, F.; Bufon, C. C. B.; Hermannstädter, C.; Witzany, M.; Beirne, G. J.; Michler, P.; Schmidt, O. G. Towards Deterministically Controlled InGaAs/GaAs Lateral Quantum Dot Molecules. New J. Phys. 2008, 10, 045010.

11. Atkinson, P.; Kiravittaya, S.; Beyoucef, M.; Rastelli, A.; Schmidt, O. G. Site-Controlled Growth and Luminescence of InAs Quantum Dots Using in Situ Ga-Assisted Deoxidation of Patterned Substrates. Appl. Phys. Lett. 2008, 93, 101908-1-3.

12. Schneider, C.; Straub, M.; Sünner, T.; Huggenberger, A.; Wiener, D.; Reitzenstein, S.; Kamp, M.; Höfling, S.; Forchel, A. Lithographic Alignment to Site-Controlled Quantum Dots for Device Integration. Appl. Phys. Lett. 2008, 92, 183101-1-3.

13. Martín-Sánchez, J.; González, Y.; González, L.; Tello, M.; García, R.; Granados, D.; García, J. M.; Briones, F. Ordered InAs Quantum Dots on Pre-Patterned GaAs (001) by Local Oxidation Nanolithography. J. Cryst. Growth 2005, 284, 313-318.

14. Kim, J.; Kawabe, M.; Koguchi, N. Ordering of High-Quality InAs Quantum Dots on Defect-Free Nanoholes. Appl. Phys. Lett. 2006, 88, 072107-1-3.

15. Song, H. Z.; Usuki, T.; Ohshima, T.; Sakuma, Y.; Kawabe, M.; Okada, Y.; Takemoto, K.; Miyazawa, T.; Hirose, S.; Nakata, Y.; Takatsu, M.; Yokoyama, N. Site-Controlled Quantum Dots Fabricated Using an Atomic-Force Microscope Assisted Technique. Nanoscale Res. Lett. 2006, 1, 160-166.

16. Martín-Sánchez, J.; González, Y.; Alonso-González, P.; González, L. Improvement of InAs Quantum Dots Optical Properties in Close Proximity to GaAs (001) Substrate Surface. J. Cryst. Growth 2008, 310, 4676-4680.

17. Martín-Sánchez, J.; Alonso-González, P.; Herranz, J.; González, Y.; González, L. Site-Controlled Lateral Arrangements of InAs Quantum Dots Grown on GaAs (001) Patterned Substrates by AFM Local Oxidation Nanolithography. Nanotechnology 2009, 20, 125302.

18. Briones, F.; Ruiz, A. Atomic Layer Molecular Beam Epitaxy (ALMBE): Growth Kinetics and Applications. J. Cryst. Growth 1991, 111, 194-199.

19. Egilsson, T.; Gislason, H. P.; Yang, B. H. Pasivation of Copper by Lithium in P-Type GaAs. Phys. Rev. B 1994, 50, 1996-1998.

20. Rodt, S.; Seguin, R.; Schliwa, A.; Guffarth, F.; Pötschke, K.; Pohl, U. W.; Bimberg, D. Size-Dependent Binding Energies and Fine-Structure Splitting of Excitonic Complexes in
Single InAs/GaAs Quantum Dots. J. Lumin. 2007, 122-123, 735-739.

21. Muñoz-Matutano, G.; Alén, B.; Martínez-Pastor, J.; Seravalli, L.; Frigeri, P.; Franchi, S. Selective Optical Pumping of Charged Excitons in Unintentionally Doped InAs Quantum Dots. Nanotechnology 2008, 19, 145711.

22. Wang, C. F.; Badolato, A.; Wilson-Rae, I.; Petroff, P. M.; Hu, E.; Urayama, J.; Imamoglu, A. Optical Properties of Single InAs Quantum Dots in Close Proximity to Surfaces. Appl. Phys. Lett. 2004, 85, 3423-3425.

23. Feucker, M.; Seguin, R.; Rodt, S.; Hoffmann, A.; Bimberg, D. Decay Dynamics of Neutral and Charged Excitonic Complexes in Single InAs/GaAs Quantum Dots. Appl. Phys. Lett. 2008, 92, 063116-1-3.

24. Hanbury-Brown, R.; Twiss, R. Q. The Question of Correlation Between Photons in Coherent Light Rays. Nature 1956, 178, 1447-1448.

25. Koashi, M.; Kono, K.; Hirano, T.; Matsuoka, M. Photon Antibunching in Pulsed Squeezed Light Generated via Parametric Amplification. Phys. Rev. Lett. 1993, 71, 1164-1167.

26. Santori, C.; Fattal, D.; Vuckovic, J.; Solomon, G. S.; Yamamoto, Y. Single-Photon Generation with InAs Quantum Dots. New J. Phys. 2004, 6, 89. 